\title{
A Study Paper : Novel Approaches for Invasive and Non-Invasive Vagus Nerve Stimulation
}

\author{
Suyog Pathare ${ }^{1}$, Supriya Mangalpalli ${ }^{2}$ \\ ${ }^{1}$ Student, Vidyalankar Institute of Technology \\ ${ }^{2}$ Teaching Assistant, Vidyalankar Institute of Technology
}

\begin{abstract}
The vagus nerve stimulation non-invasively is an approved therapy for the treatment of adult patients and adolescents and which is used to treat few neurological disorders such as epilepsy, major depressive disorder, schizophrenia, depression etc. The vagus nerve is $10^{\text {th }}$ cranial nerve (longest nerve) connected to major parts of body. The electrical stimulation from vagus nerve stimulator/generator is passed through electrodes/leads placed around vagus nerve near neck to decrease high excitation of brain which is caused due to imbalance in electrical and chemical activity in brain. The stimulator consists of microcontroller/programmer used for controlling timing and intensity of electrical pulses provided to brain to avoid seizers. The decrease/control in excitation of brain reduces possibilities of seizer attacks in patients to avoid patients from facing hazardous effects due to continuous/abnormal seizers. Hence this study is to compare efficacy and reliability of devices used as vagus nerve stimulator.
\end{abstract}

Keywords: Epilepsy, Biomedical Measurement, Biomedical Monitoring, Brain Stimulation

\section{Introduction(Epilepsy)}

Epilepsy creates problem with the brain's electrical system. Electrical impulses to the brain cause changes in movement, behavior, feeling, or awareness. These seizures may last from a few seconds to a few minutes. People having more seizures without obvious triggers separated by at least 24 hours are considered to have epilepsy. Seizures can trigger a wide range of other symptoms from staring to falling to fumbling with clothes.

Psychobiology and psychopharmacology developments have provided considerable results that major depressive disorder and bipolar depression are considered as biological disease. The previous techniques were deep brain stimulation to avoid sudden seizers but since it was inefficient to provide stimulation at the proper time of seizers that technique was overridden by Vagus Nerve Stimulation. In particular, abnormally low concentrations of serotonin and its metabolites have been found in depressed patients.

The Neurological disorders such as seizers, epilepsy, schizophrenia, heart-rate variability etc. is caused due to disruption in normal brain circuitry because of minor head injuries, stroke, oxygen deprivation, brain infection or disease etc. Doctors divide seizures into different types depending on how the brain is affected such as absence seizers (patient at once stops whatever he is doing and stares vacantly for a few seconds and will continue as nothing happened), Tonic-Clonic seizers (patient experiences with a stiffening of the arms and legs, and are followed by jerking motions), Partial seizers (patient having a simple partial seizure may have jerking motions or hallucinations, and still the patient will be aware of what is happening).

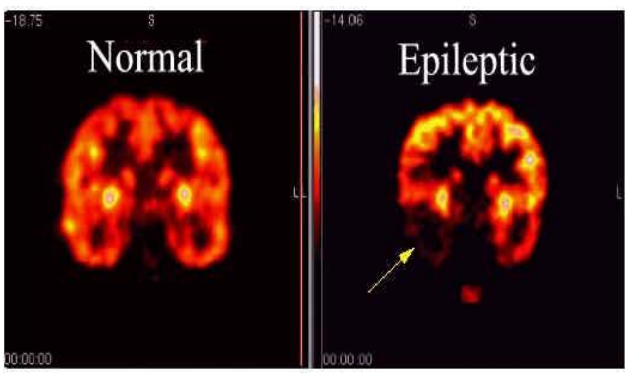

Figure 1: Thermograph of Brain

The vagus nerve stimulator is used when patient does not respond to medications and any other treatment. Ways to avoid neurological disorders are neurological stimulation, or surgery to remove affected part of the brain and medications. The neurons in the brain communicate with organs of body by sending electrical signals in a proper pattern. This pattern can be disrupted by any genetic disorder or head injury which causes uneven/high excitation of brain.

\section{Comparison with available Ideas of Vagus Nerve Stimulation}

Vagus nerve stimulation (VNS) stimulates brain with mild pulses of electrical energy via the vagus nerve, through a device similar to a pacemaker. The physical involvement of the brain in this surgery is zero and patients hardly feel the pulses. The VNS is a treatment limited to selected individuals with epilepsy or treatment-resistant depression, it is very important to remember this.

1. Invasive Vagus Nerve Stimulator : In 1991 Joachim F. Wernicke et al. had done the primary invention was Invasive Vagus Nerve Stimulator method and apparatus for treating or controlling medical, psychiatric or neurological disorders by application of modulating electrical signals to a selected nerve or nerve bundle of the patient, and more particularly to techniques for treating patients with neuropsychiatric disorders by application of such signals to the vagus nerve, using an 


\section{International Journal of Science and Research (IJSR) \\ ISSN (Online): 2319-7064 \\ Index Copernicus Value (2013): 6.14 | Impact Factor (2014): 5.611}

implantable neurostimulating device. The invention is directed toward treating the symptoms of neuropsychiatric disorders such as schizophrenia, depression, and borderline personality disorder, by selective modulation of vagus nerve activity stimulus generator 10 includes a battery (or set of batteries) 12, which may be of any> reliable long-lasting type conventionally employed for powering implantable medical electronic devices (such as batteries employed in implantable cardiac pacemakers or defibrillators).

Device Description: In the preferred embodiment of the stimulus generator, the battery is a single lithium-thionyl chloride cell. The terminals of the cell 12 are connected to the input side of a voltage regulator 13 . The regulator smoothes the battery output to produce a clean, steady output voltage, and provides enhancement thereof such as voltage multiplication or division if necessary for a specific application. Regulator 13 supplies power to logic and control section 15, which includes a microprocessor and controls the programmable functions of the device. Among these programmable functions are output current, output signal frequency, output signal pulse width, output signal on-time, output signal off-time, daily treatment time for continuous or periodic modulation of vagal activity, and output signal-start delay time. Such programmability allows the output signal to be selectively crafted for application to the stimulating electrode set to obtain the desired modulation of vagal activity for treatment and control of the disorder. Timing signals for the logic and control functions of the generator are provided by a crystal oscillator 16 . A magneticallyactuated reed switch 14 may be incorporated in the electronics package to provide the generator with manual activation capability (by use of an external magnet, not shown, placed immediately adjacent to the package or its implant site). Built-in antenna 17 enables communication between the implanted stimulus generator and the external electronics (including both programming and monitoring devices) to permit the device to receive programming signals for parameter changes.

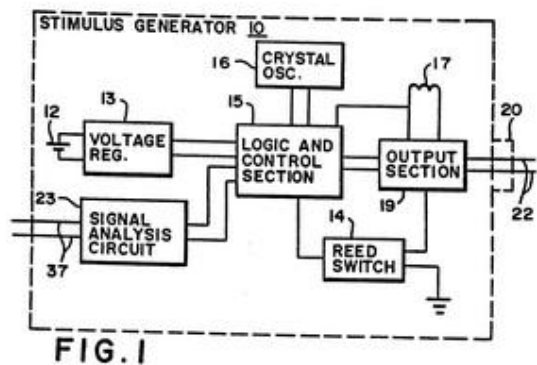

Figure 3: Internal Circuit

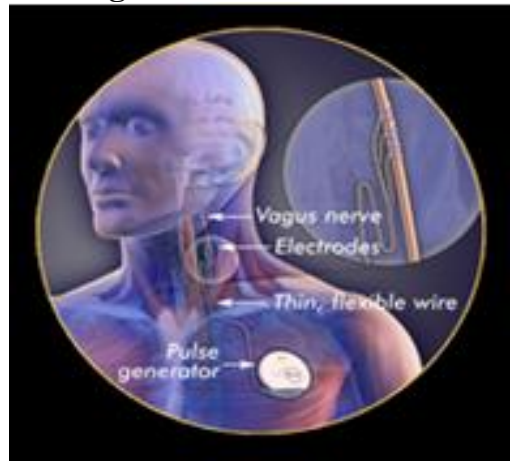

Figure 4: Setup of Implantable VNS

2. Transcutaneous Vagus Nerve Stimulator (t-VNS):Jiliang Fang et al. in (2005) next device invented was Transcutaneous Vagus Nerve Stimulator which is placed near points located in the auricular area where there are rich vagus nerve branch distributions. The device was stimulating electrical pulses through skin. It consists of electrodes which includes neuro-stimulating unit for proper pulse generation and stimulation of vagus nerve fibers which directly treats the Major Depressive Disorder, Heart rate variability etc. Transcutaneous Vagus Nerve Stimulation Modulates Default Mode Network in Major Depressive Disorder.

Working: The transcutaneous vagus nerve stimulator NEMOS consists of a stimulation unit and a dedicated ear electrode. The stimulation unit, having approximately the size of a common mobile phone, sends out the electrical impulses. It is connected with the ear electrode; which patients wear like an earphone. The impulses are transferred via the ear electrode through the skin to a branch of the vagus nerve.

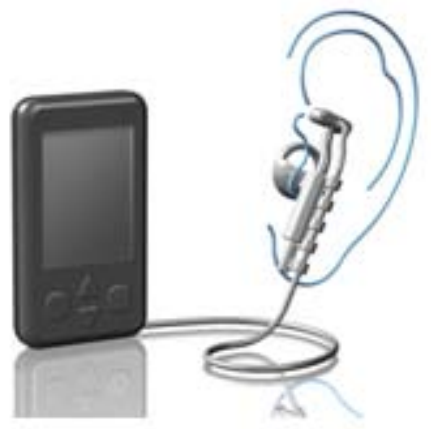

Figure 5: T-VNS (device named as NEMOS)

3. Non-Implantable Vagus Nerve Stimulator: The disclosed device was introduced to assure proper positioning of device for appropriate stimulation of vagus nerve to improve applications of the device which was not provided by tVNS. The disclosed embodiments allow patient to self-treat his or her condition. GammaCore is a portable handheld device that transmits electrical impulses to the vagus nerve without direct contact but through the skin on the right or left side of the patient's neck. The device is designed to be easy to use it is turned on and off with a simple switch and allows the patient to control the intensity of the stimulation themselves.

One dose of VNS therapy delivered by gammaCore is equivalent to approximately 90 seconds of stimulation, with each device preloaded with 50,150 , or 300 doses. For prophylactic use against cluster headache, patients are recommended to administer two doses in the morning and two doses in the evening. Based on this prophylactic protocol alone, each gammaCore device would last about 2-3 months. To treat acute episodes, patients could deliver one to three additional doses of VNS therapy in succession at the onset of pain. Each dose is only 90 


\section{International Journal of Science and Research (IJSR) \\ ISSN (Online): 2319-7064}

Index Copernicus Value (2013): 6.14 | Impact Factor (2014): 5.611

seconds but the effects of the treatment have been found to last 5-8 hours.

Working:The basic embodiment consists of chargeable or rechargeable source of electric power and two or more electrodes (345) for stimulating target nerve. Impulse generator is responsible to generate impulses for each of the electrodes (345). Control unit (330) consists of inbuilt one or more CPU for storage of programs, disk storage devices, USB for transmission and reception of data, for communication (for accepting external signals i.e. from keyboard, mouse etc.), busses for interconnection, analog to digital convertors for digitization of analog signals. (350) consists of electrically conducting medium in which electrodes are immersed. (345) It consists of Mylar sheath or any dielectric medium to act as an electrically permeable membrane.

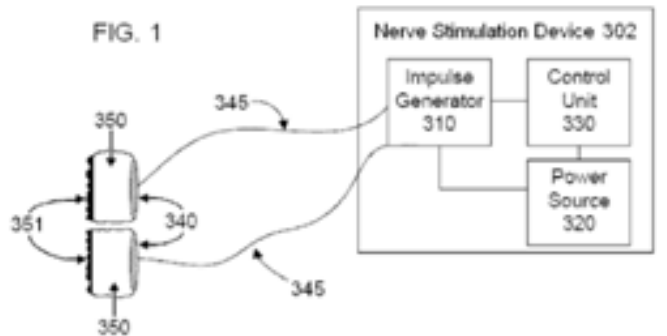

Figure 6: Internal circuit diagram

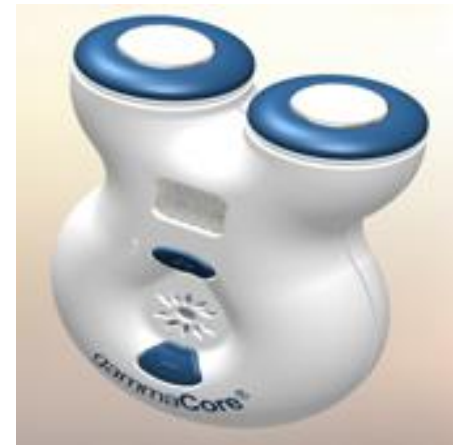

Figure 7: Implantable VNS (device named as Gammacore)

\section{Conclusion}

Research is going on, on the vagus nerve stimulator which will consist automatic stimulation of vagus nerve based on abnormal behavior of electrical impulses in brain. Add-on for asthma is also planned in the many of the above devices.

Despite the availability of multiple medications for the acute treatment of migraine attacks, patients are dissatisfied with standard pharmacologic therapy and express interest in nonpharmacologic treatments such as nVNS. The clinical benefit and ease of use of nVNS led to treatment persistence efficacy and ease of use of nVNS suggests patient satisfaction with therapy and extends previously reported findings. The efficacy of nVNS reported by patients surveyed may lessen their reliance on pharmacologic therapy, as was reported in a recent study of chronic CH13. As an effective non-pharmacologic treatment alternative for migraine, nVNS may lower the risks of medication overuse and drug-related side effects.

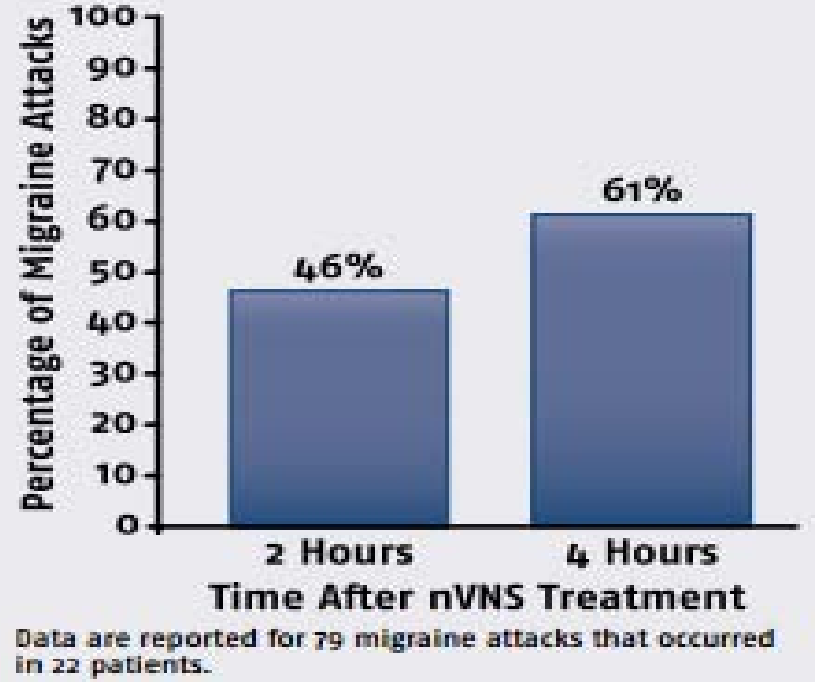

\section{References}

[1] Kristina Herbst, Birgit Sander, Dan Milea, Henrik LundAndersen and Aki Kawasaki "Test-retest repeatability of the pupil light response to blue and red stimuli in normal human eyes using a novel pupillometer", PUBMED.gov

[2] Giselle L Ferrari, Jefferson LB Marques, Rajiv A Gandhi, Simon R Heller, Fábio K Schneider, Solomon Tesfaye, and Humberto R Gamba: "Using dynamic pupillometry as a simple screening tool to detect autonomic neuropathy in patients with diabetes",PMC, US national library of medicine.

[3] Ko ML, Chen YY, Ouyang Y, Huang TW, Tsuen BS, Jeng WD, Chiou JC, Ou-Yang M."Design and analysis of wearable pupillometer for autonomic neuropathy of diabetic pateint",PUBMED.gov

[4] "Pupil monitoring can detect Diabetic nerve damage",http://blog.visivite.com/2014/08/01/pupilmonitoring-device-can-detect-diabetic-nerve-damage/

[5] http://patient.info/doctor/autonomic-neuropathy

[6] Joel Lindsey,"Wearable Device Could Provide Early Detection Of Diabetes-Related Complication", $30^{\text {th }}$ July 2014.

[7] Pei-Jing Rong et al. "Transcutaneous vagus nerve stimulation for the treatment of depression: a study protocol for a double blinded randomized clinical" triahttps://www.scienceopen.com/document_file/d8cf6e c3-5d02-4600-a5eb-

498bc6db5ec5/PubMedCentral/d8cf6ec3-5d02-4600a5eb-498bc6db5ec5.pdf.

[8] Joachim F. Wernicke et al. "Treatment of neuropsychiatric disorders by nerve stimulation" https://www.google.com/patents/US5299569.

[9] "A Visual Guide to Epilepsy", http://www.webmd.com/epilepsy/ss/slideshow-epilepsyoverview.

[10] Patent by Bruce J. Simon et al. "Devices and methods for monitoring non-invasive vagus nerve stimulation" http://www.freepatentsonline.com/20130245486.pdf.

[11] http://www.aans.org/patient\%20information/conditions \%20and\%20treatments/vagus\%20nerve\%20stimulation. aspx. "Vagus Nerve Stimulation" by AANS

[12] http://www.worldscientific.com/doi/abs/10.1142/S01290 65709001938."Non-Invasive Computerized System For Automatically Initiating Vagus Nerve Stimulation 


\section{International Journal of Science and Research (IJSR) \\ ISSN (Online): 2319-7064}

Index Copernicus Value (2013): 6.14 | Impact Factor (2014): 5.611

Following Patient-Specific Detection Of Seizures Or Epileptiform Discharges" by ALI SHOEB et al.

[13]D. Moscato, F.R. Moscato ,"A Survey of Patient Perceptions of Non-invasive Vagus Nerve Stimulation (nVNS) Therapy for Acute Migraine Attacks" http://www.ecorelibrary.com/landing/pdf/EC1558_Mosc ato_IHC_2015_Poster_WEB.pdf.

\section{Author Profile}

Suyog Pathare is with Vidyalankar Institute of Technology (affiliated to University of Mumbai), India. He is now pursuing bachelor's degree with the Department of Biomedical Engineering, Vidyalankar Institute of Technology, India.

Supriya Mangalpalli is with the Electronics Engineering Department, Vidyalankar Institute of Technology (affiliated to University of Mumbai), India, as a teaching assistant. 